\title{
Design and Build Flotocombines and Water Combines Based on the Principle of Biosimile
}

\author{
Ksenofontov B.S. (BMSTU, MOSCOW, RU)
}

\begin{abstract}
In the article offered to the attention of readers, for the first time in the world literature, both the theoretical foundations for creating flotocombines based on a multi-stage and generalized flotation model are considered, and practical recommendations based on the principles of biosimile. Possible schemes of flotocombines of the KBS type and special purpose are shown.

New aeration systems for use in various flotation apparatus, including flotocombines, are considered. Practical examples of the use of new flotation equipment are described.

It is offered to a wide range of readers, including researchers, university professors, graduate students, masters, bachelors and senior students.
\end{abstract}

KEY WORDS: waste water treatment, flotation harvesters, water harvesters, principles of biosimilarity, models of the flotation process

\section{INTRODUCTION}

The development of new equipment for wastewater treatment is due to both the need to improve the efficiency of water treatment and reduce specific material and energy costs. In this regard, there is a constant improvement of the existing and the development of new water treatment equipment [167].

For wastewater treatment, flotation equipment of mainly pressure type is used. Although the flotation process has been known for a long time, however, there are relatively few fundamentally new technical solutions in this area. As an example, we cite the method of pressure flotation developed by the author in 1989-92 with two working fluids [16], of which one is a working fluid with a hard-to-soluble gas (air), and the other with an easily soluble gas, for example, with carbon dioxide. This method was tested and used by the author for the first time in domestic practice at biotechnological enterprises.

Another example is the direction associated with the development of the use of combined plants, carried out under the leadership of the author, since 1995 at machine-building enterprises near Moscow.

The studies conducted by the author [1] determined the possibility of equipment design of this method using a combined installation, called a flotocombine by the author. The author has developed other methods and devices for flotation treatment of wastewater, taking into account their specific composition.

The direction of flotation cleaning developed by the author using multi-stage and generalized models using flotocombines developed by the author is at the stage of active implementation since the 90 s of the last century. The current stage of development of this direction is associated with the expansion of the introduction of varieties of flotocombines.

The proposed monograph examines the results of research for more than thirty years, starting from 1987 [1, 13]. At the same time, the main attention is paid to the theoretical foundations of the flotation process based on multi-page and generalized models, the development and implementation of flotocombines and water combines for both general and special purposes.

Basics of creating flotocombines using multi-layered and generalized models and principles of biosimile

The development of new flotation equipment for water purification has been carried out by us over the past few decades based on the application of a multi-stage flotation model and the principles of biosimile [1-13].

The essence of this approach lies in the fact that water purification is using flotation as the basis of a new proposed technology is considered on the basis of multi-stage and generalized models of the flotation process [1-11], and the design of a water treatment plant for the implementation of this technology is proposed on the basis of biosimile, including in one hull on a single platform. The use of multistage and generalized process models indicates the feasibility of using in flotation apparatus of a number of elements, in particular separating partitions, regulating the speed of water flow inside the apparatus, filtering nets, blocks with divergent or converging plates, etc. In Fig. Figure 1 shows a schematic diagram of the simplest flotocombine ${ }^{*}$ in the form of a flotation settling tank of the simplest design, and In Fig. 2 - a 
scheme of a flotocombine with functional elements located inside. The most significant technological achievement will be the production of condensed sludge, as well as the achievement of a higher efficiency of water purification in the latter case.

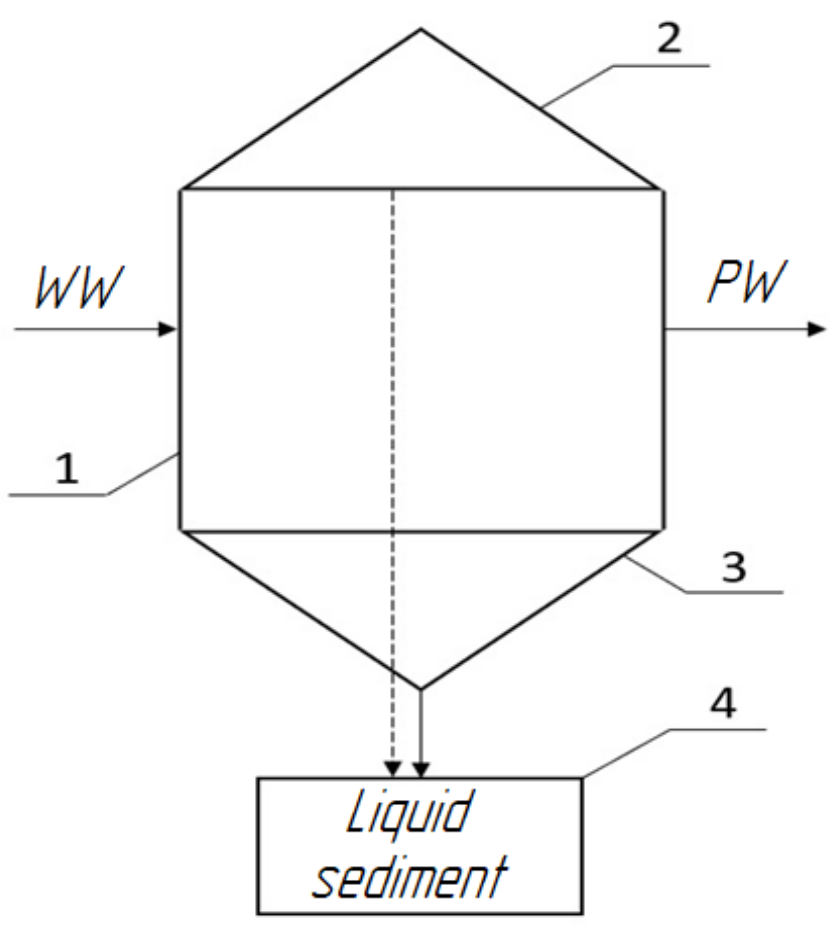

Fig. 1. Schematic diagram of the flotocombine (in the form of a flotation tank):

1 -flotocombine; 2 -foam collection zone;

3 -formation and collection of sediment; 4 - sediment collector.

$W W$-waste water; $P W$ - purified water

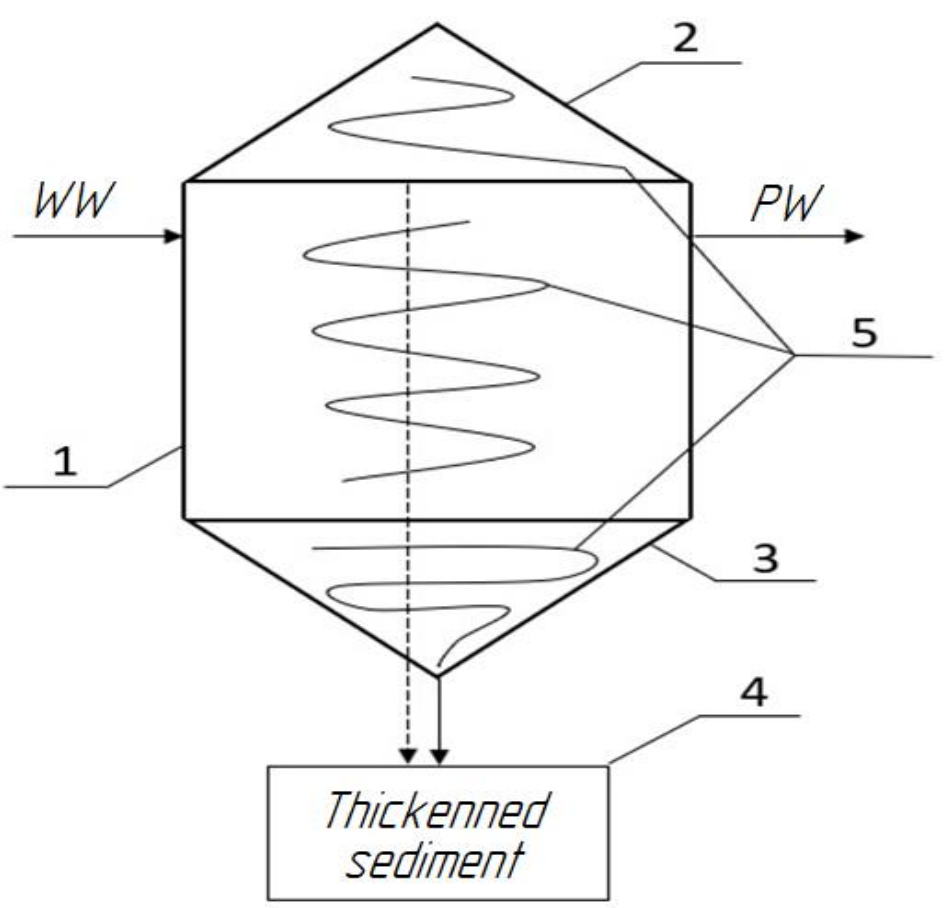

Fig. 2. Schematic diagram of the flotocombine with functional elements located inside the hull:

1 -flotocombine; 2 -foam concentrator; 3 -education zone

and condensation of sediment; 4 - condensed sludge collector; 5- Functional

elements that contribute to the intensification of water purification processes.

$W W$ - waste water; $P W$ - purged water 
An example of the implementation of the simplest version of the flotocombine in the form of a flotation settling tank is presented in Fig. 3. The main stages of the processes occurring in the workspace of such a flotation settling system are associated with the use of sedimentation, flotation and filtration.

Fig. 3. Scheme of the simplest flotocombine (flotation settling tank): 1-body of the flotocombine, 2 - a branch pipe for supplying the 1st working fluid, 3-pipes for supplying wastewater, 4 - a branch pipe for supplying a reagent solution, 5 and 7 shelves of a foam gutter, 6 - an outlet pipe for unloading foam, 8 - mesh, 9 - clarified water outlet pipe, 10 - racks, 11 bottom, 12 - sludge outlet pipe, 13 - pipe supply of the 2 nd working fluid

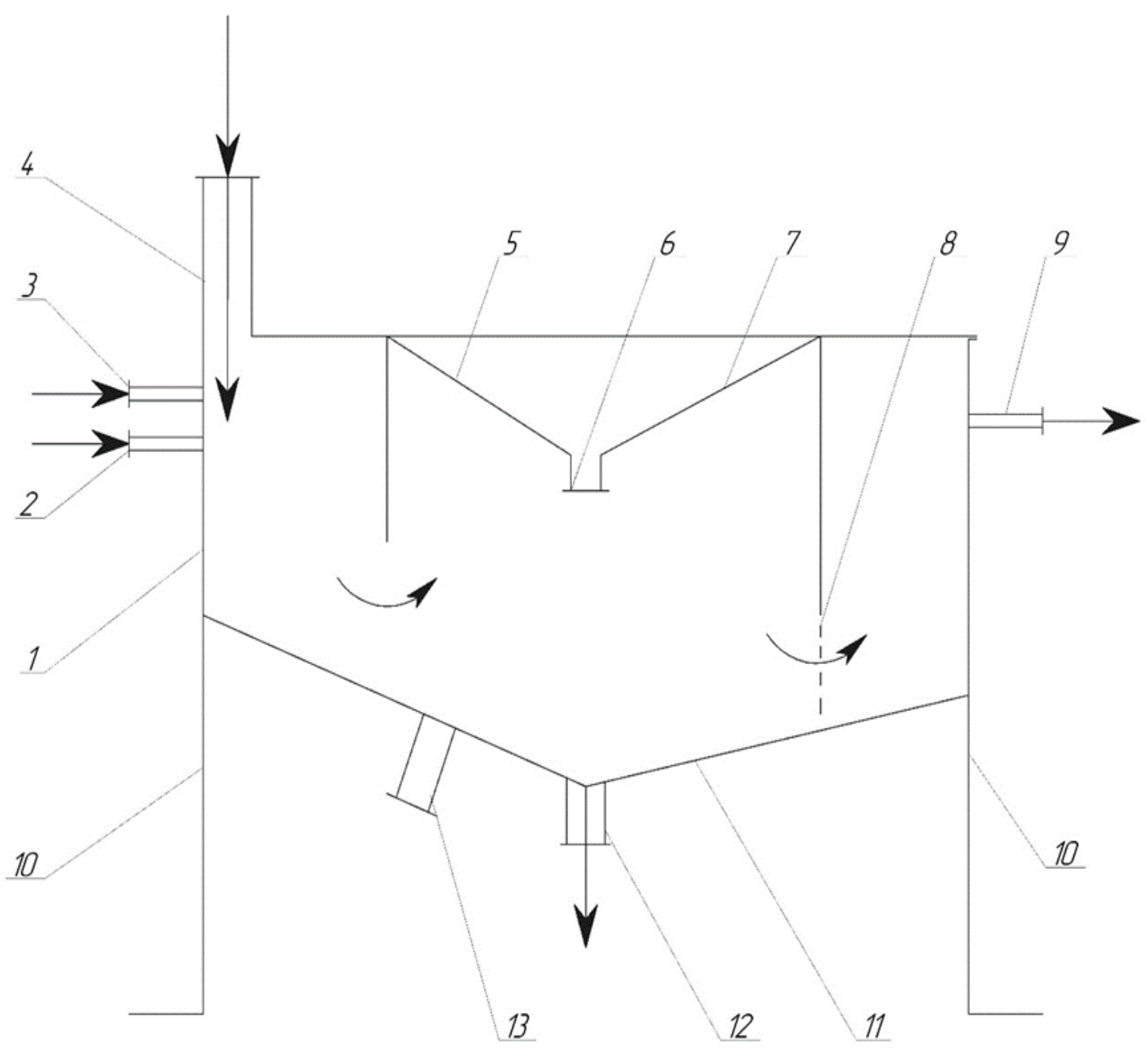

To compare the various variants of flotocombines, consider the scheme of the flotocombine presented in Fig. 4. The use of multi-stage and generalized process models indicates the expediency of using more elements in the flotocombine, in particular separating partitions, regulating the speed of water flow inside the apparatus, filtering mesh and others, as well as a block of sediment thickening (Fig. 4). 


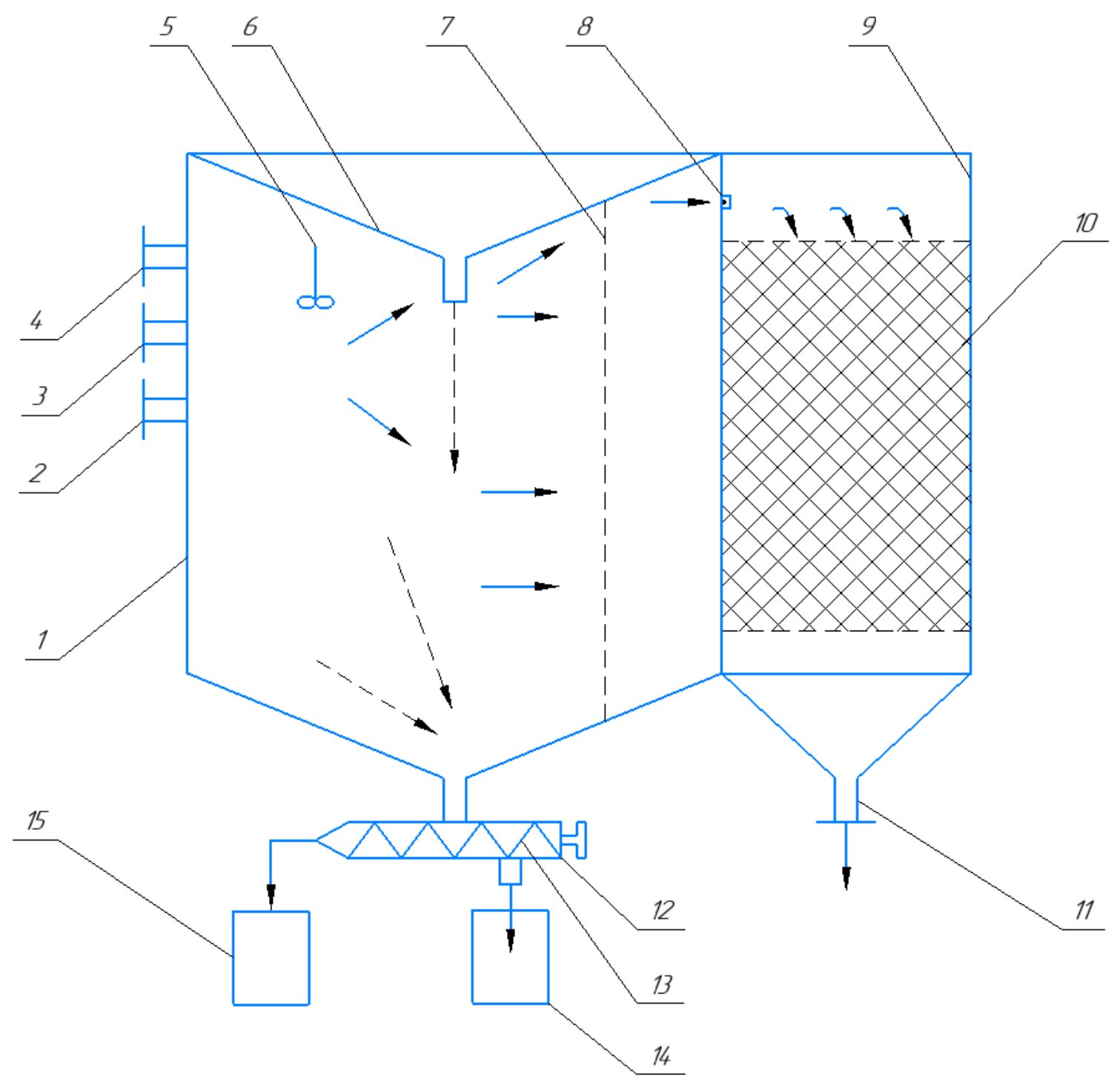

Fig. 4. Scheme of the flotocombine (author's development)

Developed by the author flotocombine (fig. 4) includes a housing 1 , on the outside of which there are branch pipes for supplying the solution 2 , the working fluid 3, the original (dirty) water 4 , the foam chute 6 , the sorption filter 9 with the filter load 10 and the outlet of pure water 11, the screw thickener 12 with the screw 13 inside connected to the collection of clarified liquid 14 and to the condensed sludge collector 15 , respectively, and inside the housing, a stirrer 5 , a mesh partition 7 and a decontaminative device 8 .

The principle of operation of the flotocombine includes the supply of dirty water through the branch pipe 4 inside the body 1 , as well as the supply of working fluid and reagent solution, respectively, through the branch pipes 3 and 2. The incoming streams within the housing 1 are mixed by a stirrer 5, which leads to the formation of a contaminant particle-gas bubble flotation complexes and their further floating into the foam layer formed in the foam chute 6 , from which it is further fed along with the sediment into the screw thickener 12. The condensed product is collected in the collection 15, and the separated liquid in the collection 14 .

The water purified in the body 1 of the flotocombine, after the separation of contaminants mainly in the form of flotation complexes, passes sequentially through the mesh partition 7, the disinfecting device 8 , for example, in the form of an ultraviolet lamp or an ozonation contact chamber, and then passes through the carbon load 10 of the filter 9 and is discharged through the branch pipe 11 .

Such a flotocombine can be autonomously used as a local treatment plant with an area smaller than in the case of using analogue facilities up to 1.6-2.2 times. 


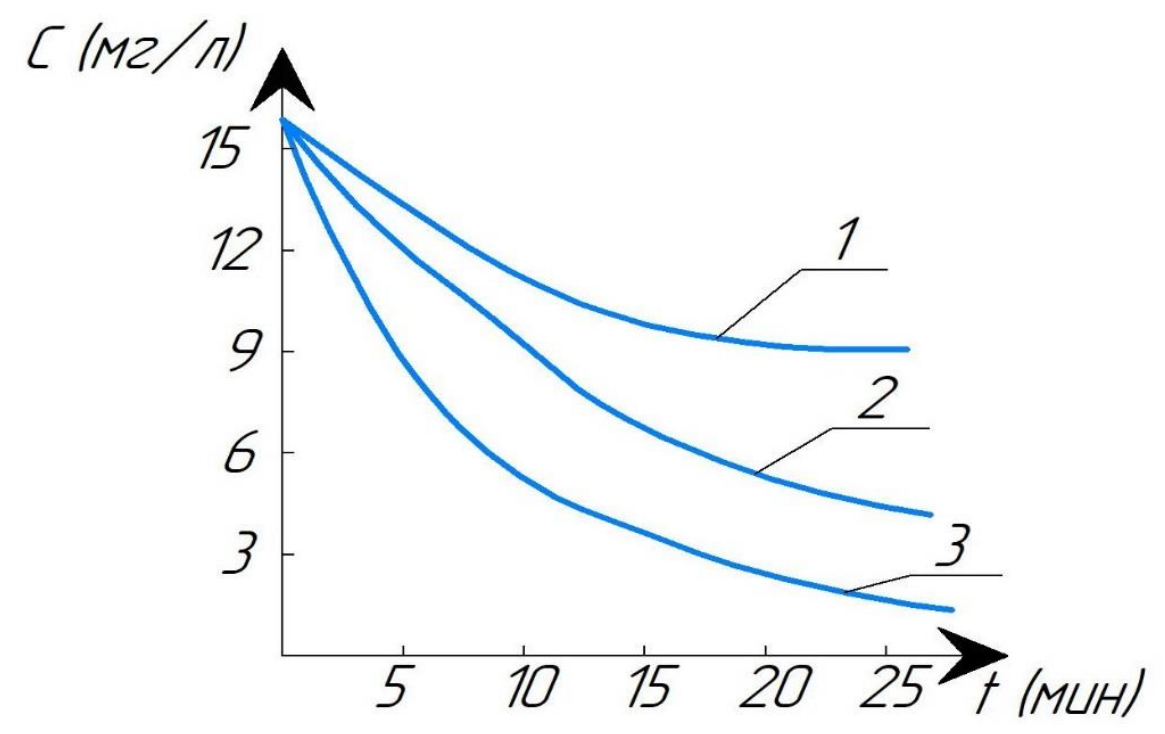

Fig. 5. Average values of the concentration of petroleum products in clarified water:

1 -after settling; 2 - after cleaning in the flotation tank;

3 -after cleaning in the flotocombine

The comparative efficiency of wastewater treatment when using different methods of treatment using, among other things, flotocombines, is shown in Fig. 5-6. It is shown that the average values of the residual concentration of petroleum products (Fig. 5) in the purified water after the flotocombine are noticeably lower than after purification in the flotation settling tank. Similar data on residual moisture content, which indicate a lower moisture content of sludge after wastewater treatment in a flocombine compared to the use of a flotation settling tank, are given in (Fig. 6).

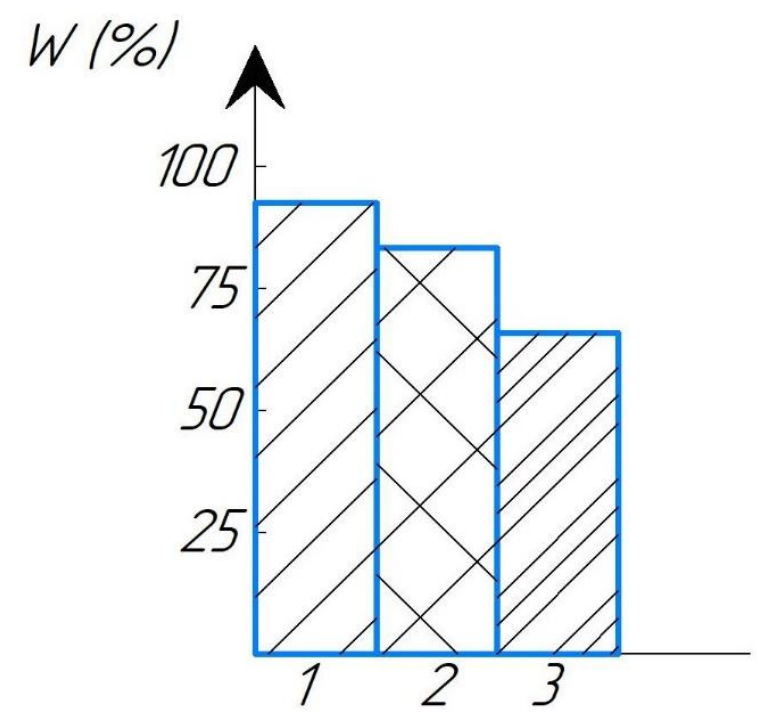

Fig. 6. Average values of moisture content of sediments formed during various types of wastewater sludge treatment: 1 -after settling; 2 - after processing in the flotation sump; 3 - after processing in the flotocombine

Thus, the treatment of wastewater in a flotocombine leads to noticeably better results than when using a flotation settling tank.

It is also important to note the use of kinetic models of cleaning processes, including flotation. A new approach based on the multi-step process proved successful not only for the intensification of processes on existing, but also on newly developed equipment [6-11]. At the same time, the development of the modeling process contributed to the emergence of a new type of equipment - flotocombines, which 
turned out to be more efficient with lower material and energy costs than well-known machines and devices.

\section{Examples of flotocombines, created using the principle of biosimile}

One of the main directions of development of flotation equipment is the creation of larger and more costeffective machines. Recently, there has been a shift from the principles of simplifying structures and mechanisms to principles that allow the separation and directing of fluid flows, the external flow of air to large installations and, thus, the use of large-volume monocamera.

The development of new flotation equipment for water purification has been carried out by us over the past few decades on the basis of the principles of biosimile and the application of a multi-stage flotation model [63]. Schematically, the concept of this approach is shown in Fig. 7.

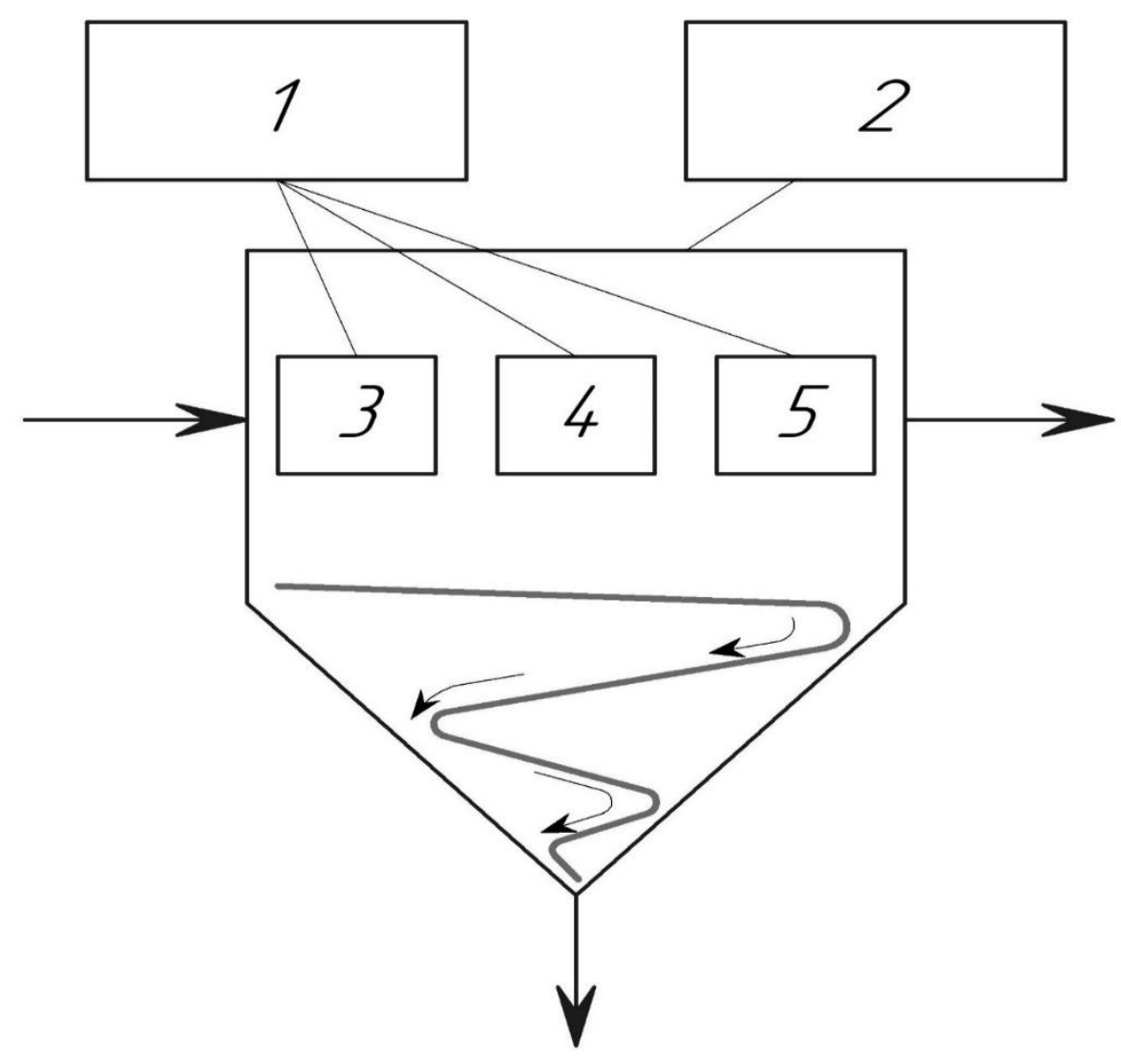

Fig. 7. Basics of the concept of biosimile and the use of modeling:

1 - multi-stage and generalized flotation models;

2 - the principle of biosimile; 3 - settling; 4 -flotation; 5 -filtration

The essence of this approach lies in the fact that water purification using flotation as the basis of a new proposed technology is considered on the basis of multi-stage and generalized models of the flotation process, and the design of a water treatment plant for the implementation of this technology is proposed on the basis of biosimile, including in one hull on a single platform. The use of multistage and generalized process models indicates the expediency of using a number of elements in the flotation apparatus, in particular separating partitions, regulating the speed of water flow inside the apparatus, filtering mesh, etc. (Fig. 8). 


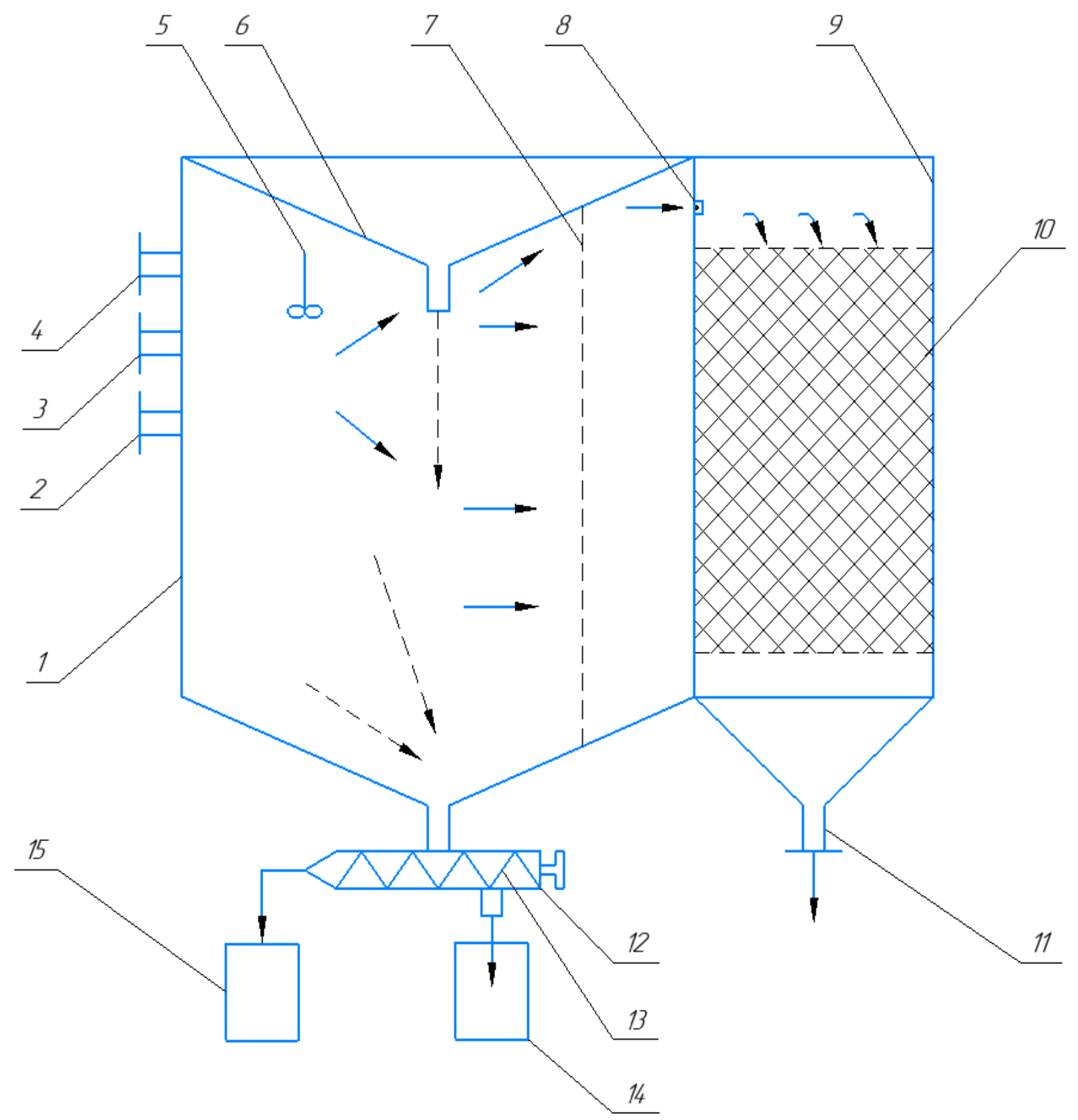

Fig. 8. Scheme of the flotocombine (author's development)

The flotocombine developed by the author (Fig. 1.8) includes a housing 1 , on the outside of which there are branch pipes for the supply of solution 2 , working fluid 3 , initial (dirty) water 4 , foam chute 6 , sorption filter 9 with filter loading 10 and a pipe of clear water outlet 11 , screw thickener 12 with an auger 13 inside connected to a collection of clarified liquid 14 and to a condensed sludge collector 15 , and inside the housing, a stirrer 5, a mesh partition 7 and a decontaminative device 8 .

The principle of operation of the flotocombine includes the supply of dirty water through the branch pipe 4 inside the body 1 , as well as the supply of working fluid and reagent solution, respectively, through the branch pipes 3 and 2. The incoming streams within the housing 1 are mixed by a stirrer 5, which leads to the formation of a contaminant particle-gas bubble flotation complexes and their further floating into the foam layer formed in the foam chute 6 , from which it is further fed along with the sediment into the screw thickener 12. The condensed product is collected in the collection 15, and the separated liquid in the collection 14 .

The water purified in the body 1 of the flotocombine, after the separation of contaminants mainly in the form of flotation complexes, passes sequentially through the mesh partition 7, the disinfecting device 8 , for example, in the form of an ultraviolet lamp or an ozonation contact chamber, and then passes through the carbon load 10 of the filter 9 and is discharged through the branch pipe 11 . The quality of treatment meets the regulatory requirements when discharging water into the city sewerage.

The next version of the flotocombine will be considered on another example, which also includes all the necessary elements of devices of this type. 
The proposed flotocombine (Figure 9) includes a separator 1 consisting of a flotation chamber 7 and a settling chamber 8 , on the outside of which are installed branch pipes for the supply of dirty water 3 and working fluid 2, the drainage of purified water 6 , the sediment 9 and the flotation sludge 5 , and inside the body in the settling chamber a screw insert 18 is installed, made of a material with a hydrophobic surface, and the branch pipe of the flotation sludge 5 is connected to the ejector 16 with the branch pipe 17, installed above the filter belt 10, above which is the sludge drainage branch pipe 9, and under the filter tape are installed liquid collectors 13 with a storage part 12 and condensed sediment 11 , and the liquid collector 13 is made in the form of a confusor, and the ejector 16 is made with an elongated mixing chamber 15 with an outlet pipe 14 and inside the housing at the inlet of the dirty water pipe a calming partition 4 is installed and at the same time the branch pipe of the sediment 9 is equipped with an adjusting valve.

The principle of operation of the flotocombine is as follows. The original dirty water enters the flotation chamber 7 of the hull 1 of the flotocombine through the branch pipe 3 . The resulting mixture of dirty water comes into contact with the working fluid entering through the nozzle 2 and containing microbubbles. The resulting flotation complexes of pollution particles - microbubbles of air rise up into the foam layer and are removed through the branch pipe 5. In this case, the non-floting contaminants enter the settling chamber 8 , in which the clarified liquid rises up and is removed from the body 1 through the branch pipe 6 , and the contaminants, rolling down the hydrophobic surface of the screw insert 18 , rush down to the sediment outlet branch pipe 9 with an adjustable valve. The hydrophobicity of the surface of the screw insert, for example, made of plastic coated with oil paint, contributes to the accelerated precipitation of contaminants.

The resulting pollution flotocomplexes - air bubbles float, creating a flotosludge in the form of a foam layer, which is removed through the branch pipe 5 and further sucked into the ejector, in which, due to the supply of a gas flow supplied through the pipe 17 from the external compressed air system, a vacuum is created. Further, in the elongated mixing chamber 15 , the flotation sludge is completely destroyed and a dirty concentrated liquid is formed, which is fed through the outlet pipe 14 to the filter belt 10 , on which contaminants in the form of both flotation and sludge discharged through a branch pipe with an adjustable valve 9 are retained and then discharged into the collector 11 , and the clarified water enters the collection of the confusory type 13 with the storage part 12.

As a result of wastewater treatment in the proposed flotation settling tank, the degree of pollution recovery reaches $95-99 \%$, while the residual moisture content is 75$85 \%$.

Thus, the proposed flotation settling tank allows for deeper wastewater treatment, in particular, to increase the degree of pollution recovery by about $10-25 \%$ and reduce the residual moisture content of the sludge by $15 \ldots 20 \%$ compared to the corresponding indicators of known naval sump.

Consider another example of the implementation of a flotocombine with the stage of condensation of the sludge by filtration.

The proposed flotocombine includes a hull 19 (Figure 1.9) inside which is a separator 1 consisting of a flotation chamber 7 and a settling chamber 8 . On the outside of the divider, branch pipes are installed for the supply of dirty water 3 and working fluid 2, the drainage of purified water 6 , sediment 9 and flotation sludge 5, and inside the separator in the settling chamber a screw insert 18 is installed, made of a material with a hydrophobic surface, and the branch pipe of the flotation sludge 5 is connected to the ejector 16 with the branch pipe 17 installed above the filter tape 10 with holders 20. At the same time, a sediment exhaust pipe 9 is located above the filter belt 10, and under this tape there are liquid collectors 13 with a storage part 12 and condensed sediment 11 , and the liquid collector 13 is made in the form of a confusor with supports 21 , and the ejector 16 is made with an elongated mixing chamber 15 with an outlet pipe 14 and inside the body at the inlet of the dirty water pipe a soothing partition 4 is installed and at the same time the sediment outlet branch pipe 9 is equipped with an adjusting valve. 


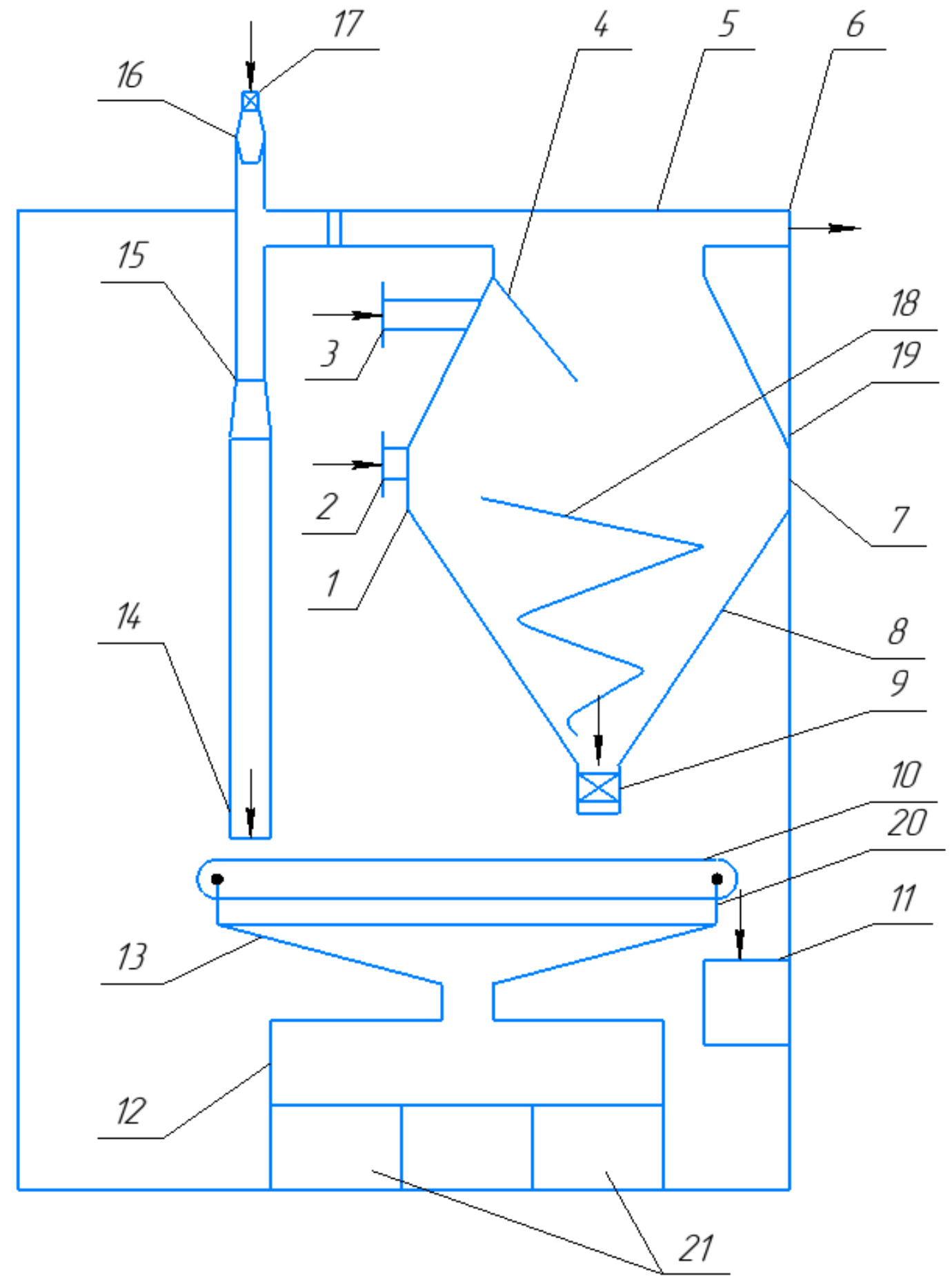

Fig. 9. Scheme of the flotocombine with a sludge filtration unit (author's development)

The principle of operation of the flotation settling system is as follows. The original dirty water enters the flotation chamber 7 of separator 1 via the branch pipe 3 . The resulting mixture of dirty water comes into contact with the working fluid entering through the nozzle 2 and containing microbubbles. The resulting flotation complexes of pollution particles microbubbles of air rise up into the foam layer and are removed through the branch pipe 5. In this case, the non-floting contaminants enter the settling chamber 8 , in which the clarified liquid rises up and is removed from the body 1 through the branch pipe 6 , and the contaminants, rolling down the hydrophobic surface of the screw insert 18 , rush down to the sediment outlet branch pipe 9 with an adjustable valve. The hydrophobicity of the surface of the screw inserts, for example, made of plastic coated with oil paint, contributes to the accelerated precipitation of contaminants.

The resulting pollution flotocomplexes - air bubbles float, creating a flotosludge in the form of a foam layer, which is removed through the branch pipe 5 and further sucked into the ejector, in which, due to the supply of a gas flow supplied through the pipe 17 from the external compressed air system, a vacuum is created. Further, in the elongated mixing 
chamber 15 , the flotation sludge is completely destroyed and a dirty concentrated liquid is formed, which is fed through the outlet pipe 14 to the filter belt 10 , on which contaminants in the form of both flotation and sludge discharged through a branch pipe with an adjustable valve 9 are retained and then discharged into the collector 11 , and the clarified water enters the collection of the confusory type 13 with the storage part 12 .

As a result of wastewater treatment in the proposed flotation settling tank, the degree of pollution recovery reaches $95-99 \%$, while the residual moisture content is $75-$ $85 \%$.

Thus, the proposed flotation settling tank allows for deeper wastewater treatment, in particular, to increase the degree of pollution recovery by about $10-25 \%$ and reduce the residual moisture content of the sludge by $15 \ldots 20 \%$ compared to the corresponding indicators of known naval sump.

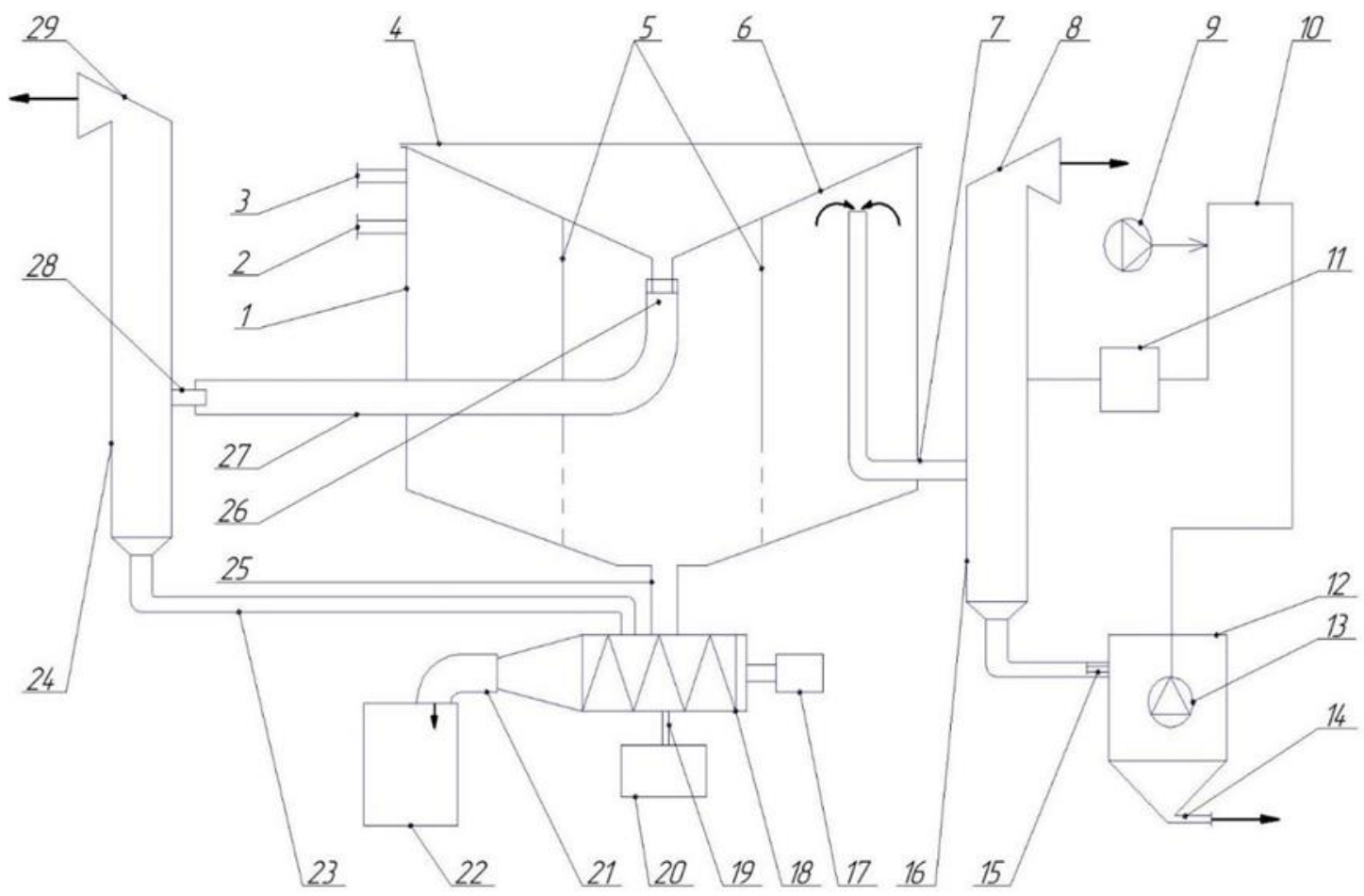

Fig. 10. Scheme of bioflot of the combine (author's development) [64]

The creation of an installation in one hull on a single platform will also be considered on the example of a bioflotcombine with a column biofilter [64]. The bioflotocombine (Fig. 1.10) includes a housing inside which partitions are located, and on the outside there are branch pipes for the supply of working fluid, wastewater, a lid, a foam gutter with a branch pipe for the extraction of the foam product, a branch pipe for the extraction of purified water, a foam product, a connecting branch pipe for the removal of sediment into a screw thickener having an external drive, and the purified water pipe is connected in series by a pipeline with a column bioreactor with loading, and the outlet of the foam chute with a flotation settling tank, the settling part of which is connected to the inlet pipe of the screw thickener, and a carbon adsorbent is used as a load, which is both stationary and fluidized.

The bioflotocombine (Figure 10.24) includes a housing 1 , inside of which partitions 5 are located, and on the outside there are branch pipes respectively for the supply of working fluid 2 , wastewater 3 , cover 4 , foam gutter 6 with a branch pipe for the outlet of the foam product 25 , a branch pipe and a purified water outlet pipe 7 connected to a column bioreactor 16 , the outlet pipe 15 of which is connected to an intermediate tank 12, having an outlet 14 on the outside at the bottom and a pump 13 connected by a conduit 10 with a tiein from the compressor 9 with an aeration chamber 11 and having a diffuser 8 at the top for the removal of gases. In this case, the branch pipe 26 of the foam chute 6 is connected by a pipeline 27 to the branch pipe 28 of the flotation settling tank 24 having at the bottom a diverter pipeline 23 connected to a screw thickener 18 with an external drive 17 , and on the outside of it there is a connecting branch pipe 25 with a body 1 for draining the sediment and an outlet pipe 19 for diverting the liquid phase to the collector 20 and the outlet pipe 21 of the concentrate into the collection 22 , and a clarified water diffuser 29 is installed at the top of the flotation settling tank 24.

The principle of operation of the proposed bioflotcombine is as follows. Wastewater through the branch pipe 3 and the working fluid through the branch pipe 2 enter the housing 1 , where a combined flotation process of water pre-treatment takes place. A further process of water 
purification takes place in a column bioreactor 16 with the supply of a recirculating stream to it, with the removal of part of the purified stream from the intermediate reservoir 12 using the submersible pump 13 and further the pipeline 10 with the supply of compressed air from the compressor 9 to it, followed by its dispersion in the aeration chamber 11 .

If it is necessary to remove water from the intermediate reservoir 12 , it is released through the branch pipe 14. The foam product formed during the combined flotation process of water purification is removed from the foam trough 6 and then enters through the branch pipe 26 and pipeline 27 and through the branch pipe 28 to the flotation settling tank 24, from which the sludge is discharged through the pipeline 23 to the screw thickener 18 , which also receives the sediment from the body 1 through the connecting branch pipe 25 and where it thickens, after which the condensed concentrate enters the sediment collector 22 and the clarified water the digest 20. Clarified water is discharged from the flotation settling system 24 through a diffuser 29 and used for its intended purpose.

The quality of the resulting clean water meets the water quality requirements of fishery reservoirs in all respects, and the degree of condensation of the sediment makes it transportable with residual humidity from 60 to $75 \%$.

Such a bioflotocombine can be autonomously used as a local treatment plant with an area smaller than in the case of using facilities - analogues up to 1.6-2.2 times.

The most typical option for creating a flotocombine, for example, for the post-treatment of dry water based on the principle of biosimile, is the version presented in Fig. 1.11.

The proposed flotocombine (Figure 11) for the aftertreatment of slush water includes a housing 1 , on the outside of which there are branch pipes 2, 3, 4 respectively for the supply of working fluid, reagent solution and source wastewater, as well as pipes 8 for the extraction of pure water, and inside the body there is a branch pipe 6 for the removal of the foam product accumulating under the conical part 5. In this case, the feed of the foam product is carried out through the branch pipe 11 together with the sediment accumulating in the conical part 10 to the screw thickener 12 containing the screw 13 connected by the outlet pipes of the clarified water 14 respectively connected to the reservoir 15 and the condensed product 18 connected to the sediment collection tank 17, installed on the bottom 16. In addition, a filter 9 is installed inside the housing 1 for water purification.

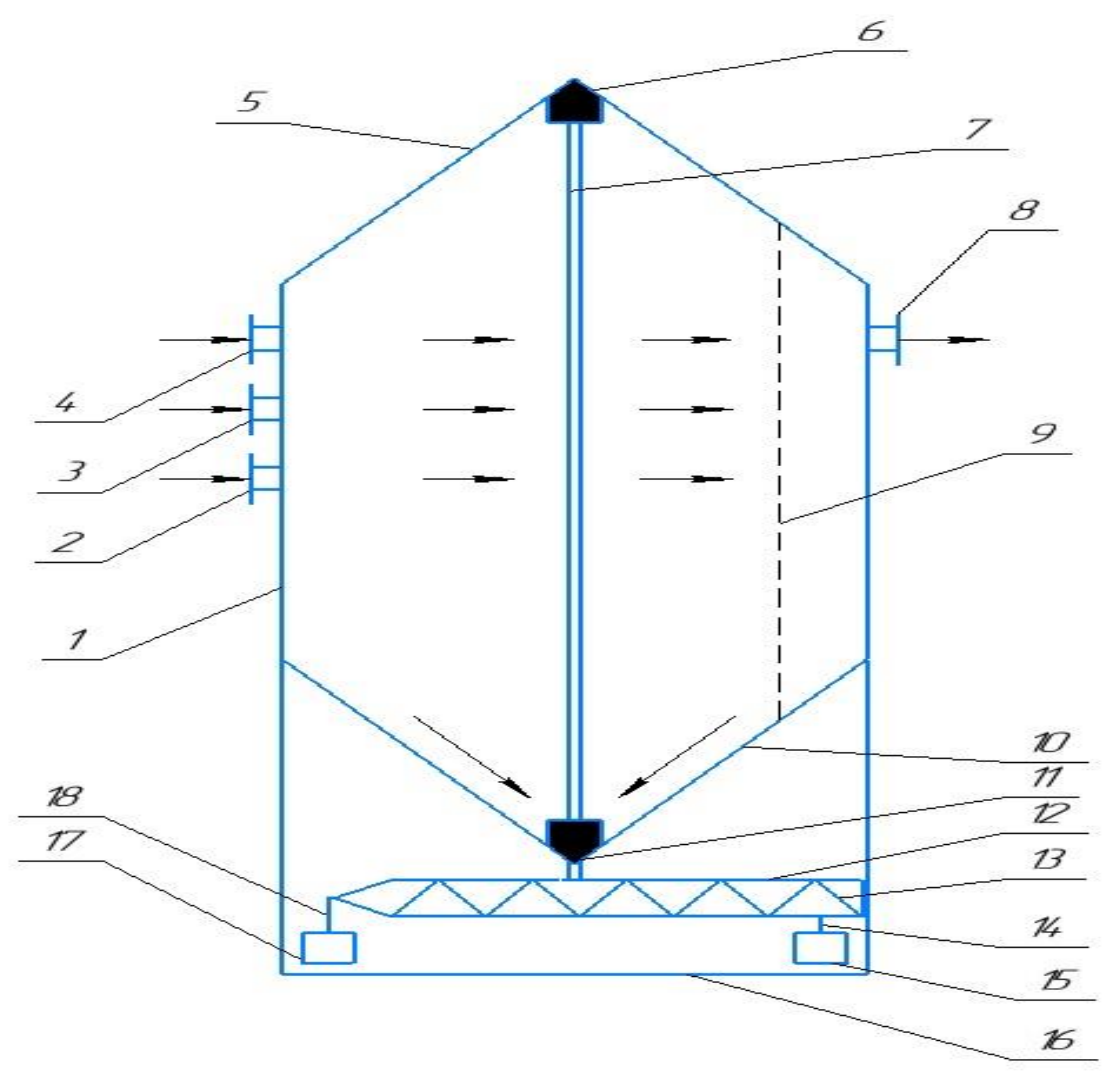

Fig. 11. Scheme of the flotocombine for sludge water treatment (author's development)

The principle of operation of the developed flotocombine is as follows. The original dirty water enters through the branch pipe 4 inside the housing 1, where the working fluid also enters through the branch pipe 2 and the solution of the reagent through the branch pipe 3. These flows are mixed, which leads to the formation of flotation complexes of pollution particle-bubble air. The flocomplexes float into a foam layer formed in the conical part 5, which is discharged 
through the branch pipe 6 and further along the pipeline 7 and further through the branch pipe 11 enter the screw thickener 12 with the screw 13, into which the sediment forming in the conical part 10 also stops. The mixture of the foam product and the precipitate is thickened and separated in the screw thickener into a clarified liquid discharged through the nozzle 14 into the reservoir 15 and the condensed concentrate through the branch pipe 18 into the reservoir 17.

The purified water is discharged through the mesh filter 9 and further through the branch pipe 8 .

The quality of treated water meets the requirements for the quality of wastewater sent to the city sewerage. At the same time, the sediment has a residual moisture content of approximately $75-85 \%$.

These examples indicate the possibility of placing separate units and devices in a single body of the flotocombine.

\section{Analogues and development concepts flotocombines and water combines}

As a natural analogue, consider an approximate mechanism for purifying water in a river. Natural water purification in flowing reservoirs is well known and described in sufficient detail in the literature. We will point out only some points, in particular the deposition of relatively large particles of the order of $0.2 \mathrm{~mm}$ or more at low water velocities in the river of the order of $0.2-0.3 \mathrm{~m} / \mathrm{s}$ (Figure 12, Pose 1). At elevated speeds, oxygen is sucked in (Fig. 12, Pos. 2 ), which further contributes to the oxidation of organic contaminants by microorganisms present in the water (Fig. 12, Pos. 3). This is roughly how water is purified in the river, if we do not consider the individual features and subtleties of those phenomena that accompany these processes. This example clearly shows that the cleaning process takes place in one body of water for a certain time. It is clear that in modern facilities, in which cleaning is carried out in stages in an intensive mode, the whole process is much faster. However, if it is possible to control the process in a reservoir like a river or pond, cleaning can be carried out within about the same time as in modern treatment facilities. For this option, it is necessary to divide the working volume into zones, for example, settling, flotation, filtration, bio-oxidation, etc. At the same time, in each zone to maintain the optimal hydrodynamic regime for this process. In this case, we have a kind of "artificial" river, with a set of nodes and elements located in it. It should be noted that the entire cleaning process is carried out in one body of a large apparatus. This leads to a significant reduction in material and energy costs. The author began the first attempts to create such devices approximately from the mid-90s of the last century. The simplest versions of such combined devices have been introduced in a number of enterprises. The author called these devices flotocombines [1], since in them the dominant position was given to the flotation process. In the future, the author began to develop devices in which flotation was not used or its use would give a slight effect compared to the overall effect achieved. The author proposed to call such combined devices water combines.

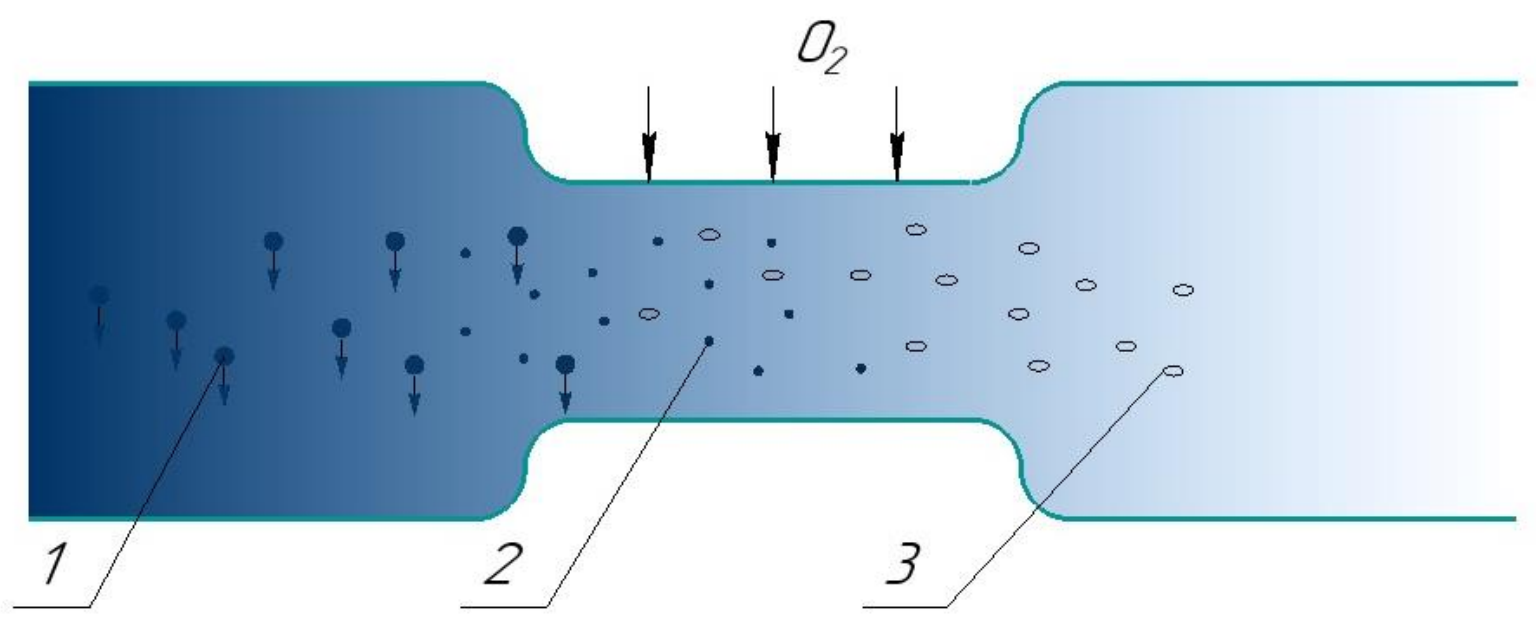

Fig. 12. Approximate mechanism of water purification in the river:

1 - coarse suspension; 2 - contaminants in the form of colloidal particles, molecules and ions; 3 -active water microorganisms

Possible schemes of the concepts for the creation of flotocombines and water combines are shown in Fig. 13 and 1.14. Presented in Fig. 13. a version of the concept of creating a flotocombine provides for a column type of apparatus. The working volume of such a floto combine is divided into several zones in which certain specific processes of water purification occur, including mechanical, physicochemical and biological methods. Wastewater treatment is carried out sequentially through the use of settling, flotation and subsequent bio-oxidation, etc. 
"Design and Build Flotocombines and Water Combines Based on the Principle of Biosimile"

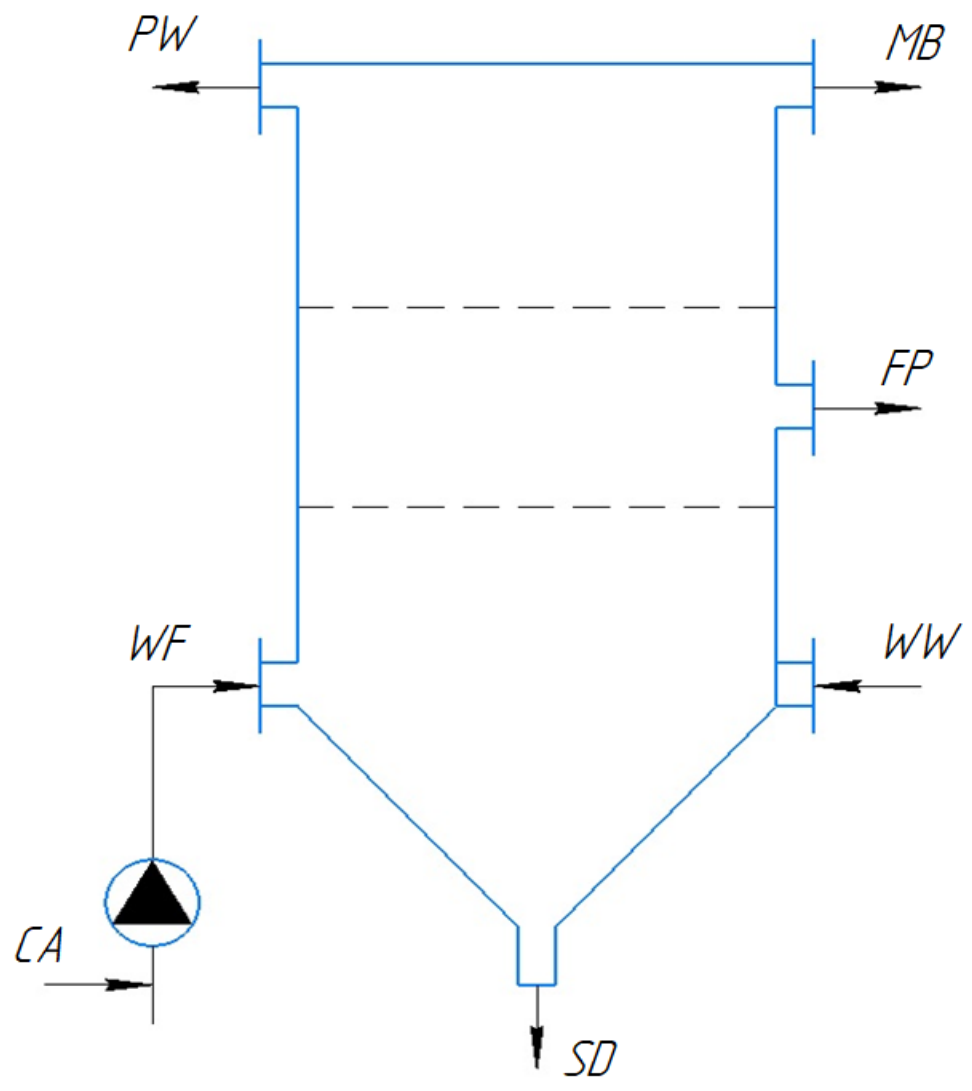

Fig. 13. Scheme of the concept of creating flotocombines using the column version: $C A$ - compressed air; $W W$ - wastewater; SD-sediment; $W F$ - working fluid; FP foam product; $P W$ - purified water; $M B$ - microbial biomass

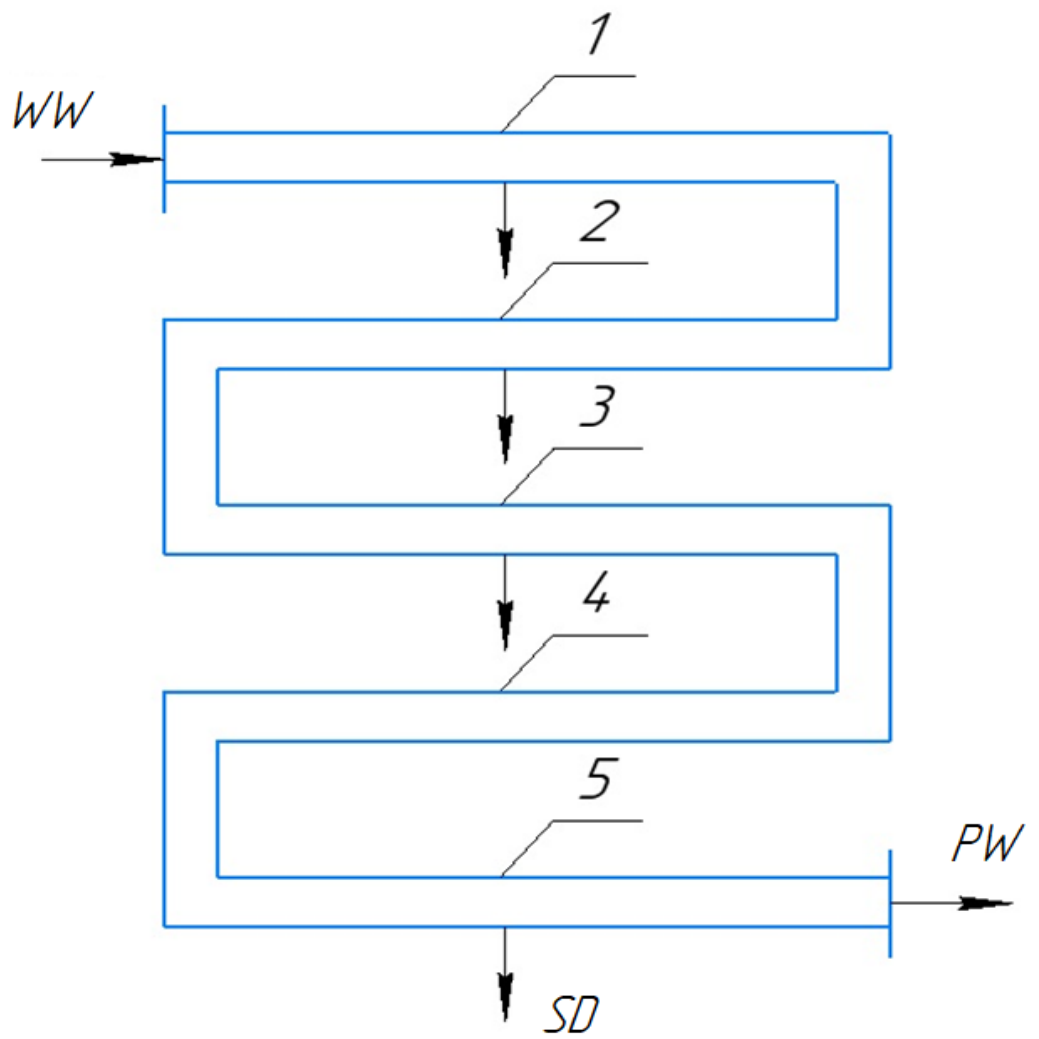

Fig. 14. Scheme of the concept of creating water combines using the channel version:

1 - sedimentation zone; 2 - contamination flotation; 3 -filtration; 
4 - bio-oxidation of contaminants; 5 - disinfection;

$W W$-wastewater; $P W$ - purified water; SD - sediment

In Fig. 14 presents a version of the water combine of the channel type. In this case, as in the case of a flotocombine, the working volume of the device is divided into a number of zones. However, the role of flotation in this option is not one of the main ones.

Analysis of the presented version of the water combine shows that in this case complete water purification is possible.

\section{REFERENCES}

1. Ksenofontov B.S. Use of multi-stadied flotation model and development of flotocombines of the CBS type for water purification. M.: Izd-vo MSTU named after N.E. Bauman. 2019. - 156 p.

2. Ksenofontov B.S. Flotation: multi-stadiy and generalized process models and flotocombines of the KBS type and special purpose. Tver: Tverskoy gos. un-t, 2020.- 424 p.

3. Ksenofontov B.S. Wastewater treatment: kinetics of flotation and flotocombines. M.: ID: «Forum»: INFRA -M. 2015. - 256 p.

4. Ksenofontov B.S., Kapitonova S.N., Senik E.V. The use of Ksenofontov's multi-stadied model in the processes of flotation wastewater treatment: monograph. Tver': Tver. State University, 2019. $162 \mathrm{p}$.

5. Patent of the Russian Federation for utility model No. 170182. Flotocombine for wastewater treatment, pr. 25.07.2016, reg. 18.04.2017. Author and applicant Ksenofontov B.S.

6. Ksenofontov B.S. Wastewater treatment in flotation columns. Water purification. 2018, NoNo1-2, p.1823.

7. Ksenofontov B.S. Mathematical models of complex articulated processes in flotocombines for wastewater treatment. Water purification. 2018, No10, p.7-11.

8. Ksenofontov B.S. Purification of industrial wastewater from petroleum products by flotation with do-extraction of microflotocomplexes. Water purification. 2018, No10, p.12-18.

9. Ksenofontov B.S. Intensification of wastewater treatment using combined flotation equipment. Water purification, 2018, No.4, p.8-13.

10. Ksenofontov B.S. Models of complex flotation processes of wastewater treatment. Water Purification, 2018, No. 6, pp. 59-69.

11. Ksenofontov B.S. Wastewater Treatment: MultiStadiy Model flotation and flotocombines. Water purification. 2018, No12, p.5-21.
12. Ksenofontov, B. S. Flotation treatment of water, waste and soil. M.: Novye ye nye nye nyi. - 2010. $272 \mathrm{p}$.

13. Ksenofontov B.S. Modeling of the process of electroflotation wastewater treatment. Express information. Sir. "Industry of mining chemical raw materials", NIITEHIM. 1987. №4. S.1-8.

14. Gvozdev V.D., Ksenofontov B.S. Treatment of industrial wastewater and utilization of sediments. M.: Khimiya, 1988. $112 \mathrm{~s}$.

15. Ksenofontov B.S., Vinogradov M.S. Use of the generalized flotation model of Ksenofontov for the calculation of water purification processes. - Tver: Tver. state university. 2019.- 185 p.

16. Ksenofontov B.S. Wastewater treatment: flotation and condensation of precipitation. M: Khimiya, 1992.- $144 \mathrm{~s}$.

17. Deryagin, B. V. Microflotatsiya: Vodoochitka, enriching, / B. V. Deryagin, S. S. Dukhin, N. N. Rulev. - M.: Khimiya, 1986. -112 p.

18. Ksenofontov B.S. Protection of the Environment: Biotechnological Foundations. M.: izd-vo «Forum»: Infra-M. 2016. -200 p.

19. Ksenofontov, B. S. /B.S. Ksenofontov. - Treatment of sewage sludge. M.: Infra - M. $2019-262$ p.

20. Ksenofontov B.S. Intensification of wastewater treatment by flotation. - Saarbrücken: LAP LAMBERT, 2012. $99 \mathrm{~s}$

21. Ksenofontov Boris. Water systems flotation treatment. Saarbrücken (Germany): LAP LAMBERT, 2011. 189 p.

22. Kolesnikov V.A., Ilyin V.I., Kapustin Yu.I., Varaksin S.O., Kisilenko P.N., Kokarev G.A. Electroflotation technology of wastewater purification of industrial enterprises / ed. V.A. Kolesnikov. - M.: Khimiya, 2007. $-303 \mathrm{p}$.

23. Bondareva G. M. Development of the electroflotation process of extraction of surfactants and motor fuels from water effluents: dissertation ... Candidate of Chemical Sciences : Moscow, 2010. $174 \mathrm{p}$.

24. Nazarov M. V., Vainshtok P. N., Voronina A. N. Preparation of sub-commodity waters for lowpermeable oil collectors by electroflotation method. Oil and Gas Business: Electronic Scientific Journal. 2014. №1 2014.

25. Patent of the Russian Federation for invention No. 2108974 "Method ofwastewatertreatment", pr.22.04.1996, reg.20.04.1998. The author and applicant is Ksenofontov B. With. 
26. Ksenofontov B.S., Senik E.V. Wastewater treatment in flotation tanks. Life safety. 2018. - №5 (209). S. 21-26.

27. Golyman A.M. Ionnaya Flotation.- M.: Bowel, 1982.- 143 c

28. Ksenofontov B.S. Flotation wastewater treatment. M.: Novye ye nye nye nyi. 2003. - 160 p.

29. Ksenofontov B.S. - Multi-stadiy model of flotation and flotocombines: monograph. Tver': Tver. state university. 2019. - 194 p.

30. Patent of the Russian Federation for utility model No183320. Ejector - mixer, pr. 16.07.2018, reg.18.09.2018. The author and applicant, B.S. Ksenofontov.

31. Ksenofontov B.S. Wastewater treatment: kinetics of flotation and flotocombines. M.: ID: «Forum»: INFRA -M. 2015. - 256 p.

32. Ksenofontov B.S., Kapitonova S.N., Senik E.V. Use of Ksenofontov's multi-stadiy model in the processes of flotation wastewater treatment: monograph. Tver': Tver. State University, 2019. $162 \mathrm{p}$.

33. Patent of the Russian Federation for utility model No170182. Flotocombine for wastewater treatment, pr. 25.07.2016, reg. 18.04.2017. Author and applicant Ksenofontov B.S.

34. Rf Patent No. 2658411. Flotocombine for wastewater treatment, pr. 11.04.2017, reg. 21.06.2018. Author and applicant Ksenofontov B.S.

35. Rf Patent No. 2669842. Flotocombine for wastewater treatment, pr. 17.11.2017, reg. 16.10.2018. Author and applicant Ksenofontov B.S.

36. Ksenofontov B.S. Wastewater treatment in flotation columns. Water purification. 2018, NoNo1-2, p.1823.

37. Ksenofontov B.S. Mathematical models of complex articulated processes in flotocombines for wastewater treatment. Water purification. 2018, No10, p.7-11.

38. Ksenofontov B.S. Purification of industrial wastewater from petroleum products by flotation with pre-extraction of microflotocomplexes. Water purification. 2018, No10, p.12-18.

39. Ksenofontov B.S. Intensification of wastewater treatment using combined flotation equipment. Water purification, 2018, No. 4, pp. 8-13.

40. Ksenofontov B.S. Models of complex flotation processes of wastewater treatment. Water Purification, 2018, No. 6, pp. 59-69.

41. Ksenofontov B.S. Wastewater treatment: multistadiy model of flotation and flotocombines. Water purification. 2018, No12, p.5-21.

42. Ksenofontov B.S. Wastewater treatment using ion flotation. Water Purification, 2018, No6, p.5-15.
43. Ksenofontov B.S., Stelmakh E.S. Ejectors as mixers for reagent water treatment. Water purification, 2018, NoNo1-2, p.70-74.

44. Ksenofontov B.S., Stelmakh E.S. Intensification of flotation wastewater treatment using jet aerators and ejectors. Water Purification, 2018, No.6, p.25-35.

45. Ksenofontov B.S. Possibilities of using pressure flotators for thickening and inactivation of biomass of activated water. Water Purification, 2018, No.4, p.14-19.

46. Ksenofontov, B.S. Flotation method of thickening of active water using carbon dioxide / B.S. Ksenofontov, A.S. Kozodaev, L.A. Dulina // 7th International Congress "Water: Ecology and Technology" (EKVATEK-2006), T.2. M. 2006. p. 830.

47. Ksenofontov B.S. Water treatment and water disposal. M.: Izd. Forum House - Infra - M. 2018. 298 p.

48. Ksenofontov B.S., Titov K. Multistage Ksenofontov model of flotation und uts. Saarbrucken: LAP LAMBERT Acad. Publ., 2019. 57 p.

49. Ksenofontov B. With. Wastewatertreatment: multitier model of flotation and flotocombines. Water purification, 2018. No12, p.5-21.

50. Ksenofontov B.S. Generalized multi-stage flotation model and development of flotocombines of the TYPE OFKBS and special purpose for water and soil purification: monograph. Tver. gos. un-t, 2019.104 s.

51. Ksenofontov B.S. Multistage model of flotation process for water purification. IOP Conference Series: Materials Science and Engineering. 2019. Vol. 492, Issue 1. - Art. No 012033. DOI: 10. 1088/1757 -899X/492/1/012033.

52. Ksenofontov B.S. Simulation of wastewater treatment in flotation machine. AIP Conference Proceedings. 2019. - Vol. 2195. - Art. No 020070.

53. DOI: 10. 1063/1.5140170.

54. Patent of the Russian Federation for utility model 123 001. Flotation machine for wastewater treatment, pr. 24.07.12, reg.20.12.12. Authors: Ksenofontov B.S., Sazonov D.V., Applicant: Bauman Moscow State Technical University.

55. Patent of the Russian Federation for utility model 143 014. Flotation machine for wastewater treatment, pr.30.12.13, reg. 10.07.14. Authors: Ksenofontov B.S., Petrova E.V., Vinogradov M.S. Applicant: Bauman Moscow State Technical University.

56. Utility model patent No. 149273. Flotation machine for wastewater treatment // Ksenofontov B. S., Antonova E. S., declared. 24.02.2014, publ 27.12.2014. 
57. Patent of the Russian Federation for utility model No. 192973 "Bioflotocombine", pr. 18.06.2019, reg. 08.10.2019. Applicant and author Ksenofontov B.S.

58. Patent for utility model No. 194987 "Himoflotokombine", pr.09.08.2019, reg. 10.01.2020. Applicant and author Ksenofontov B.S.

59. Patent for utility model No. 194985 "Electroflotocombine", pr.22.08.2019, 10.01.2020. The applicant and author, B.S. Ksenofontov.

60. Patent for utility model No. 194986 "Deminoflototocombine", pr. 16.09.2019, reg. 10.01.2020. Applicant and author Ksenofontov B.S.

61. Patent for utility model No. 195481 "Iloflotocombine", pr.25.10.2019, reg.29.01.2020. The applicant and author, B.S. Ksenofontov.

62. Patent for utility model No. 195504 "Gruntoflotocombine", pr.05.11.2019, reg. 29.01.2020. The applicant and author Ksenofontov B.S.

63. Patent of the Russian Federation for utility model No. 199049 "Vodoflototokombine", pr. 19.03.2020, reg. 11.08.2020. Applicant and author Ksenofontov B.S.

64. Ksenofontov B.S. Use of multi-stadied flotation model and development of flotocombines of the KBS type for water purification. M.: Izd-vo MSTU named after N.E. Bauman, 2019.- 156 p.

65. Application for utility model No. 2020103550 "Bioflotocombine", pr. 28.01.2020. The applicant and author, B.S. Ksenofontov.

66. Ksenofontov B.S. Chemical water treatment: monograph /B.S. Ksenofontov. - Tver: Tver State University. 2021. - 164 p.

67. Ksenofontov B.S. Purification of technogenic waters with the use of flotocombains: monograph /B.S. Ksenofontov. - Tver: Tver State University. 2020.-154 s.

68. Ksenofontov B. S. Flotation Multistage And Generalized Models Of The Process Harvesters Of Ksenofontov Type And For Special Purpose. San Francisco , Academus Publ., 2021, pt. 1, 299 p. DOI: 10.31519/0022-8 Available at:

https://academuspub.com/en/nauka/monography/22 12/view (Date of access 22.12.2021) 